\title{
Evaluación de satisfacción en la implementación de un nuevo sistema de telemedicina en el Servicio de Otorrinolaringología del Complejo Asistencial Dr. Sótero del Río (CASR) durante la pandemia de COVID-19
}

\author{
Satisfaction evaluation in the implementation of a new telemedicine \\ system in the Otorhinolaryngology Service of Dr. Sótero del Río \\ Health Center during the COVID-19 pandemicx
}

\author{
Francisco García-Huidobro N. ${ }^{1,2}$, Matías Willson E. ${ }^{1,2}$, Valeria Sepúlveda C. ${ }^{3}$, \\ Soledad Palma R. ${ }^{1}$, Pablo Cabello E. ${ }^{1,2}$
}

\section{Resumen}

Introducción: Debido a la pandemia por SARS-CoV-2, las consultas presenciales y cirugías electivas fueron suspendidas o disminuidas para evitar la propagación viral hacia pacientes y personal de salud. En nuestro país se ha implementado la telemedicina como canal alternativo de atención. Debido a su reciente implementación en nuestra especialidad a nivel nacional, es importante conocer la percepción del paciente y proveedor sobre ella, con el fin de evaluar su factibilidad como nueva modalidad de atención en el futuro. Objetivo: Evaluar la satisfacción del paciente y del proveedor sobre el uso de telemedicina en Otorrinolaringología en nuestro hospital. Material y Método: Estudio prospectivo observacional. Se adaptaron dos instrumentos de evaluación de satisfacción reportados en la literatura, para el paciente y proveedor de salud. Las encuestas fueron administradas luego de la consulta de telemedicina a través de correo electrónico para el paciente, y como parte de la plataforma de telemedicina para el proveedor y respondidas en forma voluntaria. Las respuestas fueron analizadas utilizando medidas de frecuencia (porcentajes). Resultados: Se recopilaron 51 encuestas de pacientes y 69 de proveedores. En los pacientes, en un 98\% la telemedicina facilitó el acceso a atención, siendo del mismo nivel que una presencial (91\%). Un 98\% volvería a usarla y la recomendaría a familiares o amigos. En los proveedores, el $98 \%$ estuvo satisfecho con la teleconsulta y $89 \%$ con la plataforma. Conclusión: La atención por telemedicina es satisfactoria para usuario y proveedor en nuestro hospital. Esta es una herramienta valiosa para complementar la atención clínica otorrinolaringológica.

Palabras clave: telemedicina, teleconsulta, satisfacción usuaria otorrinolaringología, COVID-19.
Abstract
Introduction: Due to the SARS-CoV-2 pandemic, clinical consults and elective surgeries were suspended or decreased to avoid spreading the disease to patients and health per- sonnel. In our country telemedicine has been implemented as an alternate way to obtain medical attention. Given that this attention paradigm has only been recently implemented in our specialty at the national level, it is important to know the perception of the patient and health care provider in order to evaluate its feasibility as a new modality of care in the future. Aim: To assess the patients and healthcare providers satisfaction regarding the use of telemedicine in the Otorhinolaryngology Department. Material and Method: Prospec- tive observational study. Two satisfaction surveys previously reported in the literature were

${ }^{1}$ Departamento de Otorrinolaringología, Complejo Asistencial Dr. Sótero del Río. Santiago, Chile.

${ }^{2}$ Departamento de Otorrinolaringología, Pontificia Universidad Católica de Chile. Santiago, Chile.

${ }^{3}$ Escuela de Medicina, Pontificia Universidad Católica de Chile. Santiago, Chile.

Los autores declaran no tener conflictos de interés.

Recibido el 5 de septiembre de 2020. Aceptado el 6 de noviembre de 2020 .

Correspondencia: Pablo Cabello E.

Servicio de

Otorrinolaringología Centro Asistencial Dr. Sotero del Río Avenida Concha y Toro 3459, Puente Alto. Santiago, Chile. Email: Pacabell@gmail.com 
adapted for the patient and health provider. The surveys were sent after each telemedicine consultation via email to the patient, and as part of the telemedicine platform for the provider and answered voluntarily. The responses were analyzed using proportions. Results: answered surveys were obtained from 51 patients and 69 healthcare providers. In 98\% of patient's telemedicine facilitated access to care, which was found to be as good as a clinical consult in $91 \%$ of the telehealth visits. 98\% would use it again and recommend it to family or friends. In $98 \%$ of providers were satisfied with the teleconsultation and $89 \%$ with the platform. Conclusion: teleconsultation is satisfactory for both patients and providers in our hospital. This new attention modality is a valuable tool to complement the standard otolaryngologist clinical assessment.

Keywords: telemedicine, teleconsultation, patient satisfaction, otolaryngology, COVID-19.

\section{Introducción}

La telemedicina se define como la transmisión de información y servicios relacionados a la salud por medio de la tecnología de telecomunicaciones ${ }^{1}$. Esta herramienta es ampliamente utilizada a nivel mundial, y ha sido reconocida como válida y útil para entregar atención médica básica y de especialidad a pacientes en áreas rurales o con menor acceso a consulta especializada ${ }^{1-3}$.

Actualmente se han descrito dos estrategias para su realización: sincrónica y asincrónica ${ }^{4}$. La telemedicina sincrónica es una evaluación interactiva, generalmente vía videoconferencia, que busca recrear la interacción médico-paciente de una consulta presencial. Por otro lado, la evaluación asincrónica es una consulta diferida ("store-and-forward"), donde toda la información relevante del paciente, incluyendo imágenes y exámenes de laboratorio, es enviada al especialista y es revisada de forma independiente ${ }^{4}$.

En Chile, la telemedicina comienza a desarrollarse aproximadamente desde 1993, con un proyecto piloto de la Universidad Católica en conjunto con el Complejo Asistencial Dr. Sótero del Río (CASR). Este proyecto buscó desarrollar métodos óptimos de colaboración a distancia y la comparación entre el diagnóstico tradicional versus el telediagnóstico, dando inicio al Programa Nacional de Telesalud 5 .

Tras el brote del nuevo coronavirus SARSCoV-2 (síndrome respiratorio agudo severo por coronavirus tipo 2), muchos servicios clínicos de nuestro país han disminuido el número de consultas y cirugías electivas para evitar la propagación de esta pandemia. Una de las especialidades más afectadas ha sido otorrinolaringología debido a que el principal reservorio del virus es la vía aérea superior e inferior y que los procedimientos asociados a la especialidad producen gran aerosolización ${ }^{6-8}$. En este escenario, el uso de la telemedicina como nuevo paradigma de atención es considerado como una alternativa viable, satisfactoria y confiable para evaluar a pacientes con patologías asociadas al área otorrinolaringológica, reduciendo el riesgo de contagio ${ }^{9-13}$.

Actualmente, ya existen servicios de otorrinolaringología que han adoptado a la telemedicina como una opción de atención, sin embargo, a la fecha no se cuenta con reportes formales sobre su implementación ni niveles de satisfacción usuaria.

\section{Objetivo}

El presente estudio tiene como objetivo evaluar la satisfacción del paciente y del proveedor sobre el uso de telemedicina en la especialidad de otorrinolaringología en el CASR como nuevo modelo de atención durante la pandemia de COVID-19.

\section{Material y Método}

\section{Diseño del estudio}

La presente investigación fue aprobada por el Comité Ético-Científico del Servicio de Salud Metropolitano Suroriente. Se realizó un estudio prospectivo observacional donde se incluyó a pacientes atendidos por médicos pertenecientes al Servicio de Otorrinolaringología del CASR mediante la modalidad teleconsulta entre el 6 y 22 de mayo de 2020. Los pacientes incluidos en el estudio debían cumplir con criterios de inclusión para ser atendidos por 
telemedicina, definidos previamente en nuestro servicio:

1) Disponibilidad de acceso a videollamada (acceso a internet y un nivel de manejo de tecnología suficiente para conectarse por videollamada).

2) Tipo de consulta: nueva consulta o control de paciente que ya ha sido visto con anterioridad en el servicio.

3) Presentar un motivo de consulta que no requiera examen físico perentorio ni realización de un procedimiento diagnóstico inmediato, o bien contar con atenciones registradas previamente en nuestra ficha clínica electrónica. En el caso de que la atención fuera una nueva consulta, se consideraron derivaciones por diagnósticos de SAHOS, rinitis alérgica y disfonía entre otras. Sin embargo, si la interconsulta mencionaba que el paciente presentaba otorrea, disfonía prolongada con sospecha de cáncer u otro síntoma que requiriera la realización de un examen físico, los pacientes eran citados para evaluación presencial y no eran incluidos en el estudio. Por otro lado, en el caso de tratarse de controles de pacientes ya conocidos fueron considerados aquellos donde el examen físico ya estuviera registrado en la ficha clínica y cuyo motivo de consulta fuera revisión de exámenes previamente tomados como: audiometrías, v-HIT, octavo par y exámenes preoperatorios.

Todos los pacientes que por juicio clínico o cuyo motivo de consulta requiriera una atención presencial era derivado al policlínico de choque de otorrinolaringología.

\section{Atención de telemedicina y aplicación de encuestas}

Esta atención consistió en una consulta sincrónica de 30 minutos de duración con el médico, en la que se realizó anamnesis, diagnóstico clínico cuando era factible y se entregaron indicaciones por vía correo electrónico. Posterior a la teleconsulta, los pacientes recibieron vía correo electrónico automatizado una encuesta anónima y voluntaria para evaluar su experiencia y satisfacción con la consulta por telemedicina. El proveedor por su parte recibió a través de la plataforma de telemedicina la encuesta correspondiente a la atención.

Las encuestas fueron contestadas en forma voluntaria por el paciente o bien el apoderado a cargo en caso de pacientes menores de 18 años. En el caso del proveedor, la evaluación de la consulta fue realizada en forma voluntaria después de cada una de las atenciones realizadas.

\section{Instrumentos de medición}

La encuesta del paciente consta de 10 ítems y es una adaptación de la encuesta publicada por Rimmer y cols. ${ }^{14}$. La encuesta del proveedor es una adaptación de la encuesta publicada por Glasser y cols. y está compuesta por cinco ítems ${ }^{15}$. Para la adaptación al español, ambas encuestas fueron traducidas al español y luego traducidas en forma reversa por dos hablantes nativos del idioma inglés. Ambas fueron evaluadas por un comité de expertos para determinar equivalencia semántica, idiomática, experiencial y conceptual ${ }^{16}$.

\section{Análisis de datos y estadística}

Se registraron y analizaron los antecedentes demográficos de los pacientes, el tipo de consulta (ingreso o seguimiento), la elegibilidad para permanecer en control mediante esta modalidad y si el tiempo otorgado para la consulta había sido adecuado. Además, se registraron los motivos de consulta, los cuales fueron categorizados de acuerdo a las áreas anatómicas que motivaron la consulta en: rinosinusología, otología y audiología, otoneurología, cuello y laringe, patología adenoamigdalina y otros. Los resultados de las encuestas, tanto la del paciente como la del proveedor, fueron analizadas por separado de acuerdo al total de sujetos que contestaron cada una de ellas. El análisis estadístico de los datos obtenidos se realizó con el programa SPSS 22.0, utilizando estadística descriptiva y medidas de frecuencia.

\section{Resultados}

\section{Demográficos y características de la atención}

Durante el periodo estudiado, fueron atendidos 86 pacientes, presentando en detalle las características demográficas y de la consulta en la Tabla 1. La mediana de edad de los pacientes atendidos es de 11 años (rango 1-74), predo- 
minando levemente el sexo femenino en un $51 \%$. Desde el punto de vista de la consulta, la mayoría de estas corresponden a pacientes que se encontraban en control en nuestro servicio

\begin{tabular}{|lrc|}
\hline \multicolumn{2}{|c|}{ Tabla 1. Características demográficas de pacientes } \\
\hline & $\mathbf{n}$ & $\mathbf{( \% )}$ \\
\hline Total & 86 & $(100)$ \\
\hline Edad (años) & 11 & $(1-74)^{\dagger}$ \\
\hline Sexo & & \\
Femenino & 44 & $(51)$ \\
Masculino & 42 & $(49)$ \\
Tipo de consulta & & \\
Ingreso & 18 & $(21)$ \\
Control & 68 & $(79)$ \\
Motivo de consulta & & \\
Rinosinusal & 28 & $(33)$ \\
Otología y audio & 28 & $(33)$ \\
Otoneurología & 7 & $(8)$ \\
Cuello y laringe & 3 & $(3)$ \\
Patología adenoamigdalina & 19 & $(22)$ \\
Otros & 1 & $(1)$ \\
\hline
\end{tabular}

(79\%). Un 65\% del total de sujetos atendidos fueron elegibles para continuar su atención mediante esta modalidad, mientras que el $35 \%$ restantes fueron derivados a consulta presencial de urgencia o diferida según cada caso. Además, solo en un $4 \%$ de las consultas realizadas el tiempo de atención de 30 minutos no fue suficiente para solucionar el problema del paciente, siendo necesario en estos casos extender la consulta por más del tiempo estipulado, o bien cambiar la plataforma a consulta telefónica. En relación a los motivos de consulta, la mayoría fue del área rinosinusal y otología, y audiología, ambas representando un 33\% de las teleconsultas, seguidas por patologías adenoamigdalinas con un 22\%.

\section{Encuesta del paciente}

El cuestionario de satisfacción del paciente fue contestado por 51 de los 86 sujetos atendidos $(59,3 \%)$ mediante teleconsulta y sus resultados se detallan en la Tabla 2. La mayor parte de los encuestados refirió estar satisfecho con su atención $(96 \%)$ y solo dos pacientes eligie-

\begin{tabular}{|c|c|c|c|c|c|}
\hline Pregunta, n (\%) & $\begin{array}{l}\text { Muy de } \\
\text { acuerdo }\end{array}$ & De acuerdo & $\begin{array}{l}\text { Ni de acuerdo ni } \\
\text { en desacuerdo }\end{array}$ & i En & $\begin{array}{c}\text { Muy en } \\
\text { desacuerdo }\end{array}$ \\
\hline $\begin{array}{l}\text { La telemedicina hizo más fácil obtener la } \\
\text { atención oportuna }\end{array}$ & $35(69 \%)$ & $15(29 \%)$ & $1(2 \%)$ & 0 & 0 \\
\hline La consulta de telemedicina fue fácil de realizar & $31(61 \%)$ & $18(35 \%)$ & 0 & $2(4 \%)$ & 0 \\
\hline $\begin{array}{l}\text { Recibí el mismo nivel de atención que si } \\
\text { hubiera ido al hospital }\end{array}$ & $36(70,5 \%)$ & $10(19,5 \%)$ & $5(10 \%)$ & 0 & 0 \\
\hline $\begin{array}{l}\text { El tiempo utilizado durante la atención fue } \\
\text { suficiente }\end{array}$ & $39(76 \%)$ & $11(22 \%)$ & $1(2 \%)$ & 0 & 0 \\
\hline Volvería a usar la telemedicina & $35(69 \%)$ & $15(29 \%)$ & $1(2 \%)$ & 0 & 0 \\
\hline \multicolumn{3}{|l|}{ Pregunta, n (\%) } & Sí & No & Sin respuesta \\
\hline \multicolumn{3}{|c|}{ En general, ¿se encuentra satisfecho con su atención por telemedicina? } & $49(96 \%)$ & 0 & $2(4 \%)$ \\
\hline \multicolumn{3}{|l|}{ ¿Ha utilizado la telemedicina previamente? } & $5(10 \%)$ & $45(88 \%)$ & $1(2 \%)$ \\
\hline \multicolumn{3}{|l|}{ ¿Utiliza habitualmente redes sociales? } & $48(94 \%)$ & $4(6 \%)$ & 0 \\
\hline \multicolumn{3}{|c|}{ ¿Recomendaría el uso de la telemedicina a su familia y amigos? } & $50(98 \%)$ & $1(2 \%)$ & 0 \\
\hline \multicolumn{3}{|l|}{ Pregunta, n (\%) } & $>3$ horas & 1-3 horas & $<1$ hora \\
\hline \multicolumn{3}{|c|}{$\begin{array}{l}\text { ¿Cuánto tiempo cree se ahorró con la telemedicina? (Incluyendo tiempo } \\
\text { de transporte, tiempo de espera y tiempo de la consulta) }\end{array}$} & $18(35 \%)$ & $28(55 \%)$ & $510 \%$ \\
\hline
\end{tabular}




\begin{tabular}{|c|c|c|c|c|c|}
\hline Pregunta, n (\%) & $\begin{array}{l}\text { Muy de } \\
\text { acuerdo }\end{array}$ & De acuerdo & $\begin{array}{c}\text { Ni de } \\
\text { acuerdo ni en } \\
\text { desacuerdo }\end{array}$ & $\begin{array}{c}\text { En } \\
\text { desacuerdo }\end{array}$ & $\begin{array}{c}\text { Muy en } \\
\text { desacuerdo }\end{array}$ \\
\hline $\begin{array}{l}\text { ¿El haber realizado la consulta por } \\
\text { telemedicina podría cambiar el } \\
\text { pronóstico del paciente? }\end{array}$ & $27(39 \%)$ & $33(48 \%)$ & $9(13 \%)$ & 0 & 0 \\
\hline $\begin{array}{l}\text { El proceso de toma de decisiones } \\
\text { clínicas fue logrado satisfactoriamente } \\
\text { durante la consulta }\end{array}$ & $46(67 \%)$ & $21(30 \%)$ & 0 & $2(3 \%)$ & 0 \\
\hline Pregunta, n (\%) & $\begin{array}{l}\text { Completamente } \\
\text { satisfecho }\end{array}$ & $\begin{array}{l}\text { Parcialmente } \\
\text { satisfecho }\end{array}$ & Neutral & $\begin{array}{l}\text { Parcialmente } \\
\text { insatisfecho }\end{array}$ & $\begin{array}{l}\text { Completamente } \\
\text { insatisfecho }\end{array}$ \\
\hline $\begin{array}{l}\text { ¿Qué tan satisfecho se siente con el } \\
\text { resultado de la consulta? }\end{array}$ & $56(81 \%)$ & $12(17 \%)$ & 0 & $1(1 \%)$ & 0 \\
\hline $\begin{array}{l}\text { ¿Qué tan satisfecho se sintió con la } \\
\text { plataforma de telemedicina durante } \\
\text { su última consulta? }\end{array}$ & $46(67 \%)$ & $15(22 \%)$ & $3(4 \%)$ & $3(4 \%)$ & $2(3 \%)$ \\
\hline $\begin{array}{l}\text { ¿Cómo considera el grado de } \\
\text { satisfacción del paciente durante su } \\
\text { última consulta? }\end{array}$ & $57(83 \%)$ & $11(16 \%)$ & $1(1 \%)$ & 0 & 0 \\
\hline
\end{tabular}

ron la opción sin respuesta en esta pregunta. El $88 \%$ de los pacientes atendidos indicó que esta fue la primera vez que utilizaban esta modalidad de consulta. Por otro lado, se observó que la mayoría se mostró muy de acuerdo con que la consulta por este medio fue fácil de realizar (61\%), que volverían a utilizar telemedicina y que se la recomendarían a un familiar $(69 \%$ y $98 \%$ respectivamente). Solo dos pacientes indicaron estar en desacuerdo con que la consulta de telemedicina fue fácil de realizar (4\%). Además, un 55\% de los encuestados refirió haber ahorrado entre 1 y 3 horas de trayecto utilizando esta modalidad de consulta y un $35 \%$ más de 3 horas.

\section{Encuesta del proveedor}

La encuesta de satisfacción del proveedor fue contestada en 69 de las 86 consultas realizadas por los médicos del servicio, cuyos resultados se encuentran detallados en la Tabla 3. La mayoría de los médicos tratantes se mostraron completa o parcialmente satisfechos ante las preguntas del cuestionario. Un $81 \%$ refirió sentirse completamente satisfecho con el resultado de la consulta y la gran mayoría indicó estar muy de acuerdo o de acuerdo en que la consulta cambió el pronóstico del paciente y que la toma de decisiones fue satisfactoria ( $87 \%$ y $97 \%$ respectivamente). Solo en una ocasión el proveedor refirió estar parcialmente insatisfecho con el resultado de la consulta (2\%). Además, el $67 \%$ de los proveedores manifestó estar completamente satisfecho con la plataforma utilizada para realizar la teleconsulta, y el $83 \%$ considera que el paciente se encontraba completamente satisfecho posterior a ella. Del total de encuestas, solo en cinco oportunidades los proveedores se mostraron parcial o completamente insatisfechos con la plataforma utilizada $(7 \%)$.

\section{Discusión}

Durante las últimas décadas en Chile y en el mundo se ha ido dando paso a una transformación digital en distintos niveles. En salud, este fenómeno se ha evidenciado en la digitalización de la información por medio de registros clínicos electrónicos, utilización de licencias médicas electrónicas, agendamiento de consultas por vía electrónica, canales virtuales de capacitación, por mencionar algunos. Focos de desarrollo de telemedicina como lo son el hospital digital, consultas de teleasistencia a especialistas, sesiones de telerehabilitación, 
entre otros se venían gestando hace varios años en nuestro país ${ }^{17-20}$. Sin embargo, la pandemia por SARS-CoV-2 aceleró significativamente la implementación y desarrollo de plataformas capaces de entregar atención por vía remota, con el fin de mantener la atención de salud y prevenir la exposición al virus por parte de pacientes y personal sanitario.

En el área de otorrinolaringología, el nuevo coronavirus SARS-CoV-2 ha disminuido la consulta ambulatoria y de urgencia tanto a nivel nacional como internacional, lo cual podría explicarse por la instauración de cuarentenas preventivas y las restricciones de deambulación aplicadas ${ }^{21}$. Ante este escenario la telemedicina puede ser considerada como una herramienta fiable, ya que ha demostrado ser útil en otras situaciones de emergencia ${ }^{22,23}$. En Chile, durante el 2020 y como respuesta al avance de la pandemia, se habilitó en forma extraordinaria la atención por telemedicina de 40 servicios médicos, dentro de los cuales no se cuenta la consulta médica de especialidad de otorrinolaringología ${ }^{24}$. Llama la atención, en base a la evidencia internacional, la falta de respaldo por parte de las autoridades sanitarias a facilitar el acceso y oportunidad de atención en diversas áreas durante tiempos de emergencia. Ante la incertidumbre que supone esta crisis sanitaria nos parece de alta relevancia reevaluar la posibilidad de ampliar la lista de prestaciones habilitadas para ser prestadas por vía remota, y de esta forma llegar a un mayor número de pacientes con necesidades en salud.

En nuestra cohorte, la mayoría de los pacientes correspondió a pacientes pediátricos, los cuales fueron atendidos junto a un mayor de edad a cargo. El alto nivel de satisfacción general refuerza la aplicabilidad de la telemedicina en distintos grupos etarios, incluido población pediátrica, en el cual ha tomado impulso durante la actual pandemia ${ }^{25-27}$. En relación al tipo de consulta, el 79\% de los pacientes atendidos en nuestro servicio correspondieron a controles debido a los criterios de elegibilidad utilizados, especialmente el concerniente a que no debía requerir un examen físico perentorio según el motivo de consulta, siendo más fácil de lograr en pacientes en seguimiento. Esto es similar a lo reportado en la literatura en donde la elegibilidad para teleconsulta según diagnósticos otorrinolaringológicos es de un $62 \%{ }^{12}$.
En Chile aún no existen reportes relacionados a la satisfacción de la telemedicina en otorrinolaringología (ORL), siendo este el primero en evaluarla. En otros países está descrito un alto nivel de satisfacción del paciente y del médico al usar la telemedicina en ORL ${ }^{11,14}$. En nuestro estudio se encontró un alto nivel de satisfacción tanto a nivel del usuario como del proveedor asociado al sistema de teleconsulta implementado en nuestro servicio, el cual es concordante con los reportes extranjeros. Esto es significativo en el contexto que nuestro sistema de telemedicina debió ser implementado debido a una emergencia sanitaria, siendo planificado y puesto en marcha en un tiempo de aproximadamente tres semanas. La implementación de este servicio ha permitido mantener la atención de nuestros pacientes a pesar de haber sido suspendidas gran parte de las actividades presenciales regulares en un servicio de alta demanda y con una gran población asignada.

Durante la realización del estudio, solo en dos ocasiones el proveedor se mostró insatisfecho con la teleconsulta lo cual coincide con episodios de insatisfacción con la plataforma. Esto también ha sido reportado por Glaser y cols., quienes describen que la principal fuente de disconformidad corresponde a uso de plataforma de telemedicina, lo cual es concordante con nuestros resultados, donde hasta un 7\% (5/69) de los proveedores manifestaron algún grado de insatisfacción al preguntárselos dirigidamente ${ }^{15}$.

En relación con la dificultad percibida por el paciente para realizar la consulta por telemedicina, solo dos pacientes refirieron que esta fue compleja. Esto puede explicarse debido a que ambos presentaron problemas de conexión durante la atención por lo que la consulta debió extenderse más del tiempo estipulado. En nuestro caso, esto podría ser secundario al uso de una plataforma comercial para videollamadas de tipo general, que aseguraba la seguridad adecuada del proceso, pero que debido a que no era específica para telemedicina contaba con prestaciones que podrían dificultar la implementación de la videollamada en personas con menores habilidades para el manejo de la tecnología. Esto podría solucionarse con el uso de una plataforma específica para telemedicina que sea más simple y específica. Otro factor a considerar es la sobredemanda de los servicios 
de los proveedores de internet a nivel local, debido posiblemente a la implementación de teletrabajo a nivel masivo, lo cual pudo contribuir al fracaso de la plataforma en algunas consultas. El problema con la plataforma de telemedicina ha sido abordado por otros autores como Rimmer y cols. quienes reportan en su estudio que los tiempos de espera y los aspectos tecnológicos de la telemedicina fueron los motivos de insatisfacción más citados ${ }^{14}$. La identificación y mejora de estos obstáculos tecnológicos ha demostrado ser de gran importancia, ya que se ha demostrado que al intervenir en esta área la calidad del servicio mejora y, por consiguiente, la satisfacción del usuario del proveedor ${ }^{28}$. Dado que la plataforma utilizada para la realización de las consultas no es específica para este fin, los problemas antes mencionados podrían ser solucionados con la implementación de un sistema de telemedicina formal. Además, la interfaz podría ser mejorada mediante gestiones con compañías de internet local que puedan asegurar un ancho de banda adecuado para la realización de esta tarea, entendiendo que debido a la pandemia existe una sobredemanda de este bien por la implementación de teletrabajo a nivel masivo.

Por otra parte, al estratificar la satisfacción según edad, no se presentó un efecto de "brecha generacional" asociada al uso de tecnologías en telemedicina en los participantes de este estudio, lo cual es similar a lo reportado por Bailey y cols. en EE. UU. ${ }^{29}$. Este fenómeno podría ser explicado por la masificación en el uso de tecnologías de comunicación, así como por la asistencia por parte de algún familiar durante la atención a los pacientes de mayor edad.

Una de las limitaciones de este trabajo corresponde a que se usaron instrumentos de medición no validados, los cuales permitían evaluar en forma subjetiva la satisfacción tanto usuaria como la del proveedor del servicio. Sin embargo, para fines de este estudio estas resultaron ser adecuadas para evaluar en forma primaria esta nueva modalidad implementada en nuestro hospital. Los instrumentos antes mencionados nos permitieron obtener en forma rápida y precoz una evaluación inicial sobre la percepción de nuestros pacientes acerca de esta modalidad de atención con el fin de realizar los ajustes correspondientes en su funcionamiento.

\section{Conclusión}

Podemos concluir que la atención por telemedicina recientemente implementada en nuestro servicio de ORL sería satisfactoria tanto para el usuario como para el proveedor. Sin embargo, es necesario realizar estudios cuantitativos sobre satisfacción en telemedicina para obtener una visión objetiva y comparable sobre esta modalidad de atención en nuestra especialidad a nivel nacional. La telemedicina mostró ser una herramienta valiosa para complementar la atención clínica presencial en nuestro servicio, permitiendo mantener la continuidad de la atención de patologías que no requieren una atención presencial de acuerdo con los criterios establecidos debido a la pandemia por SARS-CoV-2.

El futuro de la telemedicina en el campo de la otorrinolaringología parece promisorio, sobre todo en los tiempos actuales donde el contacto presencial está limitado por las medidas de restricción de contacto social. Sin embargo, aún existen limitaciones debido a la falta de cobertura por parte del sistema asegurador estatal lo que restringe el acceso a esta nueva herramienta para una parte significativa de nuestra población. Considerando la gran cantidad de evidencia internacional sobre este ámbito, a nuestro parecer, la telemedicina en otorrinolaringología se ha convertido en una valiosa herramienta que probablemente ha llegado para quedarse.

\section{Bibliografía}

1. Bush ML, Thompson R, Irungu C, Ayugi J. The Role of Telemedicine in Auditory Rehabilitation. Otol Neurotol. 2016;37(10):1466-1474. doi: 10.1097/ MAO.0000000000001236.

2. Rinde E, Nordrum I, Nymo BJ. Telemedicine in rural Norway. World Health Forum. 1993;14(1):71-77.

3. Constanzo F, Aracena-Sherck P, Hidalgo JP, Muñoz M, Vergara G, Alvarado C. Validation of a patient satisfaction survey of the Teleneurology program in Chile. BMC Res Notes. 2019;12(1):1-7. doi: 10.1186/ s13104-019-4358-1.

4. Saadi R, Goldenberg D, Goldenberg D. Using Technology in Global Otolaryngology. Otolaryngol Clin North Am. 2018;51(3):555-561. doi: 10.1016/j. otc.2018.01.004

5. Ministerio de Salud SDR. Programa Nacional de Telesalud En El Contexto de Redes Integradas de Servicios de Salud. 2007;1-76. 
6. Tysome JR, Bhutta MF. COVID-19: Protecting our ENT Workforce. Clin Otolaryngol. 2020:0-2. doi: 10.1111/coa.13542.

7. Lagos AE, Ramos PH, Andrade T. Protection for Otolaryngologic Surgery in the COVID-19 Pandemic. OTO Open. 2020;4(2):2473974X2093473. doi: $10.1177 / 2473974 x 20934734$.

8. Krajewska J, Krajewski W, Zub K, Zatoński T. COVID-19 in otolaryngologist practice: a review of current knowledge. Eur Arch Oto-Rhino-Laryngology. 2020;(0123456789). doi: 10.1007/s00405-020-05968-y.

9. Cho RHW, Yeung ZWC, Ho OYM, et al. Pearls of experience for safe and efficient hospital practices in otorhinolaryngology-head and neck surgery in Hong Kong during the 2019 novel coronavirus disease (COVID-19) pandemic. J Otolaryngol - Head Neck Surg. 2020;49(1):1-8. doi:10.1186/s40463-020-00427-4.

10. Seim NB, Philips RHW, Matrka LA, et al. Developing a synchronous otolaryngology telemedicine Clinic: Prospective study to assess fidelity and diagnostic concordance. Laryngoscope. 2018;128(5):1068-1074. doi: 10.1002/lary.26929.

11. Yulzari R, Bretler S, Avraham Y, Sharabi-Nov A, Even-Tov E, Gilbey P. Mobile TechnologyBased Real-Time Teleotolaryngology Care Facilitated by a Nonotolaryngologist Physician in an Adult Population. Ann Otol Rhinol Laryngol. 2018;127(1):46-50. doi: 10.1177/0003489417745089.

12. McCool RR, Davies L. Where Does Telemedicine Fit into Otolaryngology? An Assessment of Telemedicine Eligibility among Otolaryngology Diagnoses. Otolaryngol - Head Neck Surg (United States). 2018;158(4):641-644. doi:10.1177/0194599818757724.

13. Smith AC, Dowthwaite S, Agnew J, Wootton R. Concordance between real-time telemedicine assessments and face-to-face consultations in paediatric otolaryngology. Med J Aust. 2008;188(8):457-460. doi: 10.5694/j.1326-5377.2008. tb01715.x

14. Rimmer RA, Christopher V, Falck A, et al. Telemedicine in otolaryngology outpatient settingsingle Center Head and Neck Surgery experience. Laryngoscope. 2018;128(9):2072-2075. doi: 10.1002/ lary.27123.

15. Glaser M, Winchell T, Plant P, et al. Provider satisfaction and patient outcomes associated with a statewide prison telemedicine program in Louisiana. Telemed e-Health. 2010;16(4):472-479. doi:10.1089/ tmj.2009.0169.

16. Tsang S, Royse CF, Terkawi AS. Guidelines for developing, translating, and validating a questionnaire in perioperative and pain medicine. Saudi J Anaesth. 2017;11(5):S80-S89. doi: 10.4103/sja. SJA_203_17.

17. Ministerio de Salud. Hospital Digital. https://www. hospitaldigital.gob.cl/. Published 2020.

18. Flores R, Donoso R, Anguita R. Modelo de manejo en red y por telemedicina de la retinopatía diabética en dos comunas del Servicio de Salud Metropolitano Oriente. Rev Med Chile 2019;147(4):444-450. doi: 10.4067/s0034-98872019000400444.

19. González Coloma F, Sandoval Garcés M, Gedda Quiroga V, Bley Banda C. Teledermatology in Remote Parts of Chile: Experience in 4 Isolated Rural Areas. Actas Dermo-Sifiliográficas (English Ed. 2019;110(8):653-658. doi: 10.1016/j. adengl.2019.07.002.

20. Caro UP, Siqués SC, Cubillos BR, García PD. Evaluación de satisfacción frente al uso del programa de telerehabilitación Rehabitic ${ }^{\circledR}$ para terapia física en niños con parálisis cerebral del Instituto Teletón Santiago. Estudio de 3 casos. Rehabil Integr. 2012;7(1):32-39.

21. Gelardi M, Iannuzzi L, Trecca EMC, Kim B, Quaranta NAA, Cassano M. COVID-19: what happened to all of the otolaryngology emergencies? Eur Arch Oto-Rhino-Laryngology. 2020;(0123456789):2-3. doi: 10.1007/s00405-020-06046-z.

22. Ning AY, Cabrera CI, D’Anza B. Telemedicine in Otolaryngology: A Systematic Review of Image Quality, Diagnostic Concordance, and Patient and Provider Satisfaction. Ann Otol Rhinol Laryngol. 2020:3489420939590. doi:10.1177/0003489420939590.

23. Arriaga MA, Nuss D, Scrantz K, et al. Telemedicineassisted neurotology in post-Katrina Southeast Louisiana. Otol Neurotol. 2010;31(3):524-527. doi: 10.1097/MAO.0b013e3181cdd69d.

24. Chávez M. Autorizan 40 tipos de atenciones por telemedicina para evitar contactos. El Mercurio. 2020:C4.

25. Jordan V. Cochrane Corner: Coronavirus (COVID-19): remote care through telehealth. J Prim Health Care. 2020;12(2):184-185. doi: 10.1071/ HC15951.

26. Mukerji SS, Liu YC, Musso MF. Pediatric otolaryngology workflow changes in a community hospital setting to decrease exposure to novel coronavirus. Int J Pediatr Otorhinolaryngol. 2020;136(January):110169. doi: 10.1016/j. ijporl.2020.110169.

27. Wood SM, White K, Peebles R, et al. Outcomes of a Rapid Adolescent Telehealth Scale-Up During the COVID-19 Pandemic. J Adolesc Heal. 2020;67(2):172178. doi: 10.1016/j.jadohealth.2020.05.025.

28. Wootton AR, McCuistian C, Legnitto Packard DA, Gruber VA, Saberi P. Overcoming Technological Challenges: Lessons Learned from a Telehealth Counseling Study. Telemed e-Health. 2019;00(00):1-6. doi: 10.1089/tmj.2019.0191.

29. Bailey CE, Kohler WJ, Makary C, Davis K, Sweet N, Carr M. eHealth Literacy in Otolaryngology Patients. Ann Otol Rhinol Laryngol. 2019;128(11):1013-1018. doi: 10.1177/0003489419856377. 